\title{
CORRECTIONS
}

\section{Dyslipidaemia and cardiovascular risk}

In an article about dyslipidaemia and cardiovascular risk by Aidan Ryan and colleagues (BMJ 2018;360:k835, doi:10.1136/ bmj.k835) Figure 1 contained an error. The box containing the statement "Creatinine Kinase $\leq 4 \mathrm{x}$ upper limit of normal" should instead read "Creatinine Kinase $\geq 4 \mathrm{x}$ upper limit of normal." 